\title{
Stochastic sensitivity of bull and bear states
}

\author{
Jochen Jungeilges ${ }^{1,2}$ - Elena Maklakova ${ }^{2}$ Tatyana Perevalova ${ }^{2}$
}

Received: 30 April 2020 / Accepted: 8 December 2020 / Published online: 14 February 2021

(c) The Author(s) 2021

\begin{abstract}
We study the price dynamics generated by a stochastic version of a Day-Huang type asset market model with heterogenous, interacting market participants. To facilitate the analysis, we introduce a methodology that allows us to assess the consequences of changes in uncertainty on the dynamics of an asset price process close to stable equilibria. In particular, we focus on noise-induced transitions between bull and bear states of the market under additive as well as parametric noise. Our results are obtained by combining the stochastic sensitivity function (SSF) approach, a mixture of analytical and numerical techniques, due to Mil'shtein and Ryashko (1995) with concepts and techniques from the study of non-smooth 1D maps. We find that the stochastic sensitivity of the respective bull and bear equilibria in the presence of additive noise is higher than under parametric noise. Thus, recurrent transitions are likely to be observed already for relatively low intensities of additive noise.
\end{abstract}

Keywords Stochastic price process · Non-invertible maps · Non-smooth maps · Stochastic sensitivity function · Transitions between stochastic fixed points · Critical intensity

\section{Introduction}

Recent events affecting commodity and asset markets worldwide in the most drastic way once more obviate the need for analytical techniques that help us to understand how sensitive market outcomes are to variations in uncertainty. Surges of uncertainty

We would like to thank the participants of the "11th Nonlinear Economic Dynamics conference, NED 2019" for their constructive comments.

$凶$ Jochen Jungeilges

jochen.jungeilges@uia.no

1 Department of Economics and Finance, School of Business and Law, University of Agder, Servicebox 422, 4604 Kristiansand S, Norway

2 Institute of Natural Science and Mathematics, Ural Federal University, Ekaterinburg, Mira 19, Russian Federation 
in the environment into which markets are embedded might - apart from affecting the behavior of market participants - have an effect on the interaction between agents. Distortions to the process of interaction which ultimately determines the market outcome will have profound effects on the development of prices. In the context of studying a stochastic asset market, we introduce a methodology that allows us to assess the consequences of changes in uncertainty on the dynamics of an asset price process.

A considerable literature relying on nonlinear dynamic models of financial markets has deepened our understanding of the functioning of asset markets. This literature has successfully emphasized the intrinsic limitations to predicting market outcomes. Within this class, studies focussing on the functioning of financial markets with heterogenous, possibly interacting investors (Lux (1995), Brock and Hommes (1998), Böhm and Wenzelburger (2005), Chiarella et al. (2005), and Huang et al. (2010)) have expanded our insights into financial markets significantly.

The studies by Day and Huang (1990) and Huang and Day (1993) represent seminal, highly influential research efforts. Considering three types of market participants, sophisticated investors, less sophisticated traders and market makers, the authors derive an asset price process based on a one-dimensional continuous map. They show that their deterministic model generates stochastic price dynamics as well as "bear and bull markets." This work has motivated the research efforts which eventually evolved into separate strands of the literature.

For example, the research efforts by Tramontana et al. (2009), Tramontana et al. (2010), Tramontana et al. (2011), Tramontana et al. (2013) and Tramontana et al. (2014) share distinct common characteristics. They all consider models of an asset market with heterogenous (possibly interacting) agents rooted in the Day-Huang paradigm. The studies focus on asset price dynamics that is driven by a onedimensional piecewise linear map (possibly with discontinuity points). With respect to research methodology, they tend to rely on a mix of neoteric analytical and numerical techniques. Strong emphasis is put on intricate bifurcation analyses. These studies not only contribute significantly to the economics of financial markets, they also augmented our understanding of the dynamics based on piecewise linear maps with discontinuities. In particular, Tramontana et al. (2015), Sushko et al. (2015) and Panchuk et al. (2018) represent recent contributions to this type of literature which should be singled out, since they motivated our own modeling effort. In each of the models studied, some traders only enter or exit the market if the current asset price differs significantly from the fundamental value of the asset.

These research efforts produce a rich body of results, each and every one of them interesting in their own right. To survey those results in full detail is prohibitive, given the scope of this paper. Viewed from the perspective of dynamics, the literature demonstrates how the full analytical treatment of piecewise linear maps with discontinuities can be achieved. Moreover, it establishes the significance of border collision bifurcations and generates insights into conditions under which equilibria and/or cycles might or might not coexist. Economics and finance benefit from this literature since it shows how bull and bear markets may emerge endogenously, how chaotic price regimes occur and that both phenomena are robust. In addition, those efforts have made clear that both fundamentalists and chartists may contribute to destabilization. 
Three aspects consistently surface as one surveys the existing literature for dimensions on which future research should concentrate. First of all, one should allow for agents' asymmetric response around the fundamental value. Secondly, the case of nonidentical no-trade intervals of fundamentalists and chartists should be scrutinized. In addition, we find the quest to intensify the stochastic modeling effort.

Our current work can be seen as a direct response to the latter two quests. In particular, we contribute to the existing literature by considering a new variant of the original Day and Huang model. While our agents' response to deviations of the asset price from the fundamental value is still symmetric, we focus on the case in which their threshold levels for market entry (chartists) and change of trading intensity (fundamentalists) do not coincide.

The second contribution is a methodological one. Introducing various types of noise, we study the sensitivity of stable equilibria (bull and bear markets) and describe conditions under which recurrent transitions between such equilibria occur. This is achieved by relying on the stochastic sensitivity function (SSF) technique due to Mil'shtein and Ryashko (1995). The approach allows us to study the interaction between various types of noise and a piecewise linear deterministic map. The SSF technique has only recently been applied in the context of piecewise linear maps. For examples involving stochastic 1-D and 2-D maps see Belyaev and Ryazanova (2019a), Belyaev and Ryazanova (2019c), Belyaev and Ryazanova (2019b) and Nasyrova et al. (2019). Our work demonstrates that the SSF technique, which combines analytical and numerical strategies, also facilitates qualitative arguments typical for the research tradition in economics and finance.

Finally, the paper furthers our understanding of asset price dynamics in speculative markets by analyzing the dynamics generated by a deterministic map ( 5 linear pieces map with 2 discontinuities) in the presence of additive and parametric noise. We identify different types of transitions between bull and bear markets and unravel the "genesis" of these transitions. In particular, it is shown how under fixed behavioral features of fundamentalists and chartists, larger uncertainties can lead to the onset of recurrent transitions. Beyond that, we also explain how such transitions may be caused by changes in investor behavior under given levels of noise. Our numerical examples demonstrate how such recurrent transitions give rise to a bi-modal marginal distribution of the asset price. This phenomenon has only recently been established as a salient characteristic of the S\&P500 by Schmitt and Westerhoff (2017).

The stochastic asset price model is presented in Sect. 2. As an integral part of the presentation, three noise regimes are specified. Since the sensitivity analysis of the attractors of the stochastic price process is based on constructs which are associated with its deterministic skeleton, we identify the map and discuss coexisting equilibria and their basins of attraction in Sect. 3. Moreover, the subset of the parameter space is identified on which our investigation is focussed. The subsequent Sect. 4 provides an outline of the conceptual background for stochastic sensitivity analysis. The key concepts presented here are motivated, applied and discussed in the context of the stochastic price process (2) with additive noise in Sect. 5. In Sect. 6, we present the results of our sensitivity analysis for the cases of parametric noise and compare the stochastic sensitivity of price equilibria under the alternative types of noise. A discussion of our results and some concluding remarks in Sect. 7 finalize the paper. 


\section{The stochastic model}

We consider a stochastic version of a Day and Huang type asset market in which three types of market participants operate: fundamentalists, chartists and a market maker who mediates and facilitates intended transactions out of equilibrium. In our model, the asset price evolves due to the interaction between the market participants who operate in an uncertain environment. We model the price process under general environmental noise as well as in the presence of noise affecting the trading behavior of fundamentalists or chartists specifically. After some conceptual qualifications, we will establish the deterministic skeleton of the price process before we proceed to the respective stochastic specification.

Prior to motivating some detail underlying the asset price dynamics which is essentially driven by a piecewise linear map, we would like to stress that the process corresponds to a piecewise nonlinear/multiplicative asset price process. To be specific, applying the homeomorphism $\ln ()$ to this piecewise nonlinear dynamic system, we generate a piecewise linear system that is topologically equivalent to the original nonlinear one. The segments of the piecewise nonlinear map are specified such (exponential functions) that they are intrinsically linear, i.e. they become linear under the appropriate transformation (natural logarithm). Throughout the remainder of the paper, we stick to the following convention: we simply refer to the asset price and the fundamental value given the understanding that $p_{t}=\ln \left(\pi_{t}\right)$ where $\pi_{t}$ denotes the price of the asset under scrutiny, $v=\ln (f)$ represents the transform of the asset's fundamental value. Moreover, the constants $\gamma$ and $\epsilon$ represent logged threshold levels from the corresponding piecewise nonlinear/multiplicative model.

Next, we will establish the excess demand functions of the key market participants. The investors referred to as fundamentalists base their investment strategy on sophisticated and technically advanced analyses. Believing in mean reversion, they will plan to buy (sell) when the current asset price (natural logarithm of the asset price) falls short of (exceeds) the asset's future economic value, reflected by $v$, the natural logarithm of the fundamental value.

Definition 1 (Excess demand of fundamentalists)

$$
\mathscr{A}(p)= \begin{cases}\alpha_{0} \gamma-\alpha(p-v+\gamma), & p \leq v-\gamma \\ \alpha_{0}(v-p), & v-\gamma<p<v+\gamma \\ -\alpha_{0} \gamma-\alpha(p-v-\gamma), & p \geq v+\gamma\end{cases}
$$

$v \in(0,1), 0<\gamma<\min (v, 1-v), \alpha_{0} \geq 0$ and $\alpha>\alpha_{0}$.

The less sophisticated $\beta$-investors, or chartists, base their investment strategy on a simple adaptive estimate of the asset's future economic value grounded on the spread between the current asset price $p$ and the current fundamental value $v$. Those investors enter (leave) the market when the price exceeds (falls short of) the fundamental value $v$ expecting further price increases (decreases). 
Definition 2 (Excess demand of chartists) The excess demand of the chartists is given by

$$
\mathscr{B}(p)= \begin{cases}\beta(p-v), & p \leq v-\epsilon^{-} \\ \beta(p-v), & p \geq v+\epsilon^{-} \\ 0, & v-\epsilon^{-}<p<v+\epsilon^{-}\end{cases}
$$

with $v \in(0,1), 0<\epsilon^{-}<\min (v, 1-v), \beta>0$.

The parameters $\alpha_{0}, \alpha$ and $\beta$ capture the respective traders' response to a marginal variation in the asset price (trade intensity). ${ }^{1}$ We assume that an agent's trade intensity changes as the asset price leaves a neighborhood around the fundamental value. In particular, the fundamentalists trade more aggressively at price levels outside the interval $(v-\gamma, v+\gamma)\left(\alpha>\alpha_{0}\right)$, while the chartists do not trade at all in an $\epsilon^{-}$ neighborhood around $v$ and switch to an intensity of $\beta$ otherwise. Thus, we capture differences in perception of the asset's fundamental value across the types of investors in a rudimentary way. For further detail, see Jungeilges et al. (2021). In fact, the current model constitutes a simplification of the model presented in Huang and Day (1993) in which fundamentalists estimate the fundamental value as a long run estimate of the stock value, while chartists rely on a recursive estimate of the fundamental value.

In the stochastic version of the model, we allow for the possibility that the agents' trading behavior could be subject to random shocks when the asset price moves sufficiently far away from the fundamental value. In the remainder of the paper, we focus on the case of $\gamma>\epsilon^{-}$. The behavioral features captured constitute the kinks at $v \pm \gamma$ in the graph of $\mathscr{A}(p)$ and jumps at $v \pm \epsilon^{-}$in the graph of $\mathscr{B}(p)$.

A market maker, assumed to be endowed with an inventory of stocks, mediates and facilitates transactions out of equilibrium. She sets the price in response to excess demand and supply signaled by fundamentalists and chartists, following a simple linear price adjustment function

$$
p_{t+1}=p_{t}+\delta\left[\mathscr{A}\left(p_{t}\right)+\mathscr{B}\left(p_{t}\right)\right]
$$

where $\delta>0$ signifies a price adjustment parameter and $\mathscr{A}\left(p_{t}\right)$ and $\mathscr{B}\left(p_{t}\right)$ can be thought of as the orders placed, respectively, by fundamentalists and chartists. Thus, at a given price the market maker satisfies excess demand out of his inventory or accumulates inventory when there is excess supply, charging commissions on transactions between fundamentalists and chartists. Allowing for additive and parametric shocks the evolution of the asset price takes the form of a stochastic difference equation.

Definition 3 (Stochastic price process) Relying on the linear price adjustment function (1) with $\delta=1$ and Definitions 1 and 2, the asset price process can be given as

$$
p_{t+1}=p_{t}+\mathscr{A}\left(p_{t} ; \gamma, \alpha_{0}, \alpha+\varepsilon_{\alpha} \xi_{t}, v\right)+\mathscr{B}\left(p_{t}, \epsilon^{-} ; \beta+\varepsilon_{\beta} \xi_{t}, v\right)+\varepsilon_{\bullet} \xi_{t}
$$

with $p_{0} \in(0,1), \varepsilon_{\bullet}, \varepsilon_{\alpha}, \varepsilon_{\beta} \geq 0, \xi_{t} \sim N(0,1)$ i.i.d.

\footnotetext{
1 Since we consider the $\ln$-transform of the asset price $\pi_{t}$, the values $-\alpha_{0},-\alpha$ and $\beta$ give the elasticities of the piecewise excess demand functions (1) and (2) with respect to the asset price.
} 
Table 1 Noise scenarios

\begin{tabular}{lll}
\hline Case & Condition & Interpretation \\
& $\varepsilon_{\alpha}=\varepsilon_{\beta}=\varepsilon_{\bullet}=0$ & Deterministic skeleton \\
\hline 1 & $\varepsilon_{\alpha}=\varepsilon_{\beta}=0, \varepsilon_{\bullet}>0$ & Additive shock(s) \\
2 & $\varepsilon_{\alpha}>0, \varepsilon_{\beta}=0, \varepsilon_{\bullet}=0$ & Parametric shock $(\alpha)$ \\
3 & $\varepsilon_{\alpha}=0, \varepsilon_{\beta}>0, \varepsilon_{\bullet}=0$ & Parametric shock $(\beta)$ \\
\hline
\end{tabular}

Throughout this paper, we are assuming that the populations of fundamentalists and chartists differ in terms of their trading strategies as well as with respect to the threshold levels $\gamma$ and $\epsilon^{-}$.

Assumption 1 (Heterogeneity) With respect to fundamentalists and chartist, we assume (1) $\alpha>\beta+1$ and (2) $\gamma>\epsilon^{-}$.

The market is affected by an exogenous shock $\xi_{t}$ (meteorological, political, pandemic, etc.) which might influence the price process in a non-specific additive manner and/or by affecting the trading intensities of specific market participants far away from the fundamental value, i.e., when the asset traded is severely mispriced. The additive shock in this log price model corresponds to a multiplicative-exponential shock $\phi_{t}^{\varepsilon_{\bullet}}=\left(e^{\xi_{t}}\right)^{\varepsilon_{\bullet}}$, where $\phi_{t}$ follows a log-normal distribution with $\mathbb{E}\left[\phi_{t}\right]=\sqrt{e} \approx 1.6487$ and $\mathbb{V}\left[\phi_{t}\right]=e^{2}-e^{1} \approx 4.6710$ to the piecewise nonlinear multiplicative price process.

The cases singled out for analysis are listed in Table 1 . These types of specifications are not without precedence in the related literature. Examples for asset price dynamics depending on additive shocks to account for factors lying outside the realm of the model (case 1) can be found in Lux (1995), Brock and Hommes (1998), Gaunersdorfer and Hommes (2007). In those efforts, the shocks are typically modeled as i.i.d. normal random variables. Also trading rules have been subjected to noise. For example, Franke and Westerhoff (2012) rely on additive stochastic components in the trading rules for chartists as well as fundamentalists to capture the diversity of actual fundamental and technical trading rules. Closer to our cases 2 and 3 , is the specification found in Cafferata and Tramontana (2019) who model the reactivity of chartists to the price signal as a random walk involving Gaussian noise.

We study the stochastic price process with the intention to understand the nature of noise-induced transitions occurring between the most simple coexisting attractors (price equilibria). The analysis builds on the stochastic sensitivity function approach, an indirect method of studying stochastic dynamic processes which requires, at the outset, the analysis of the deterministic skeleton underlying the stochastic price process (2).

\section{The deterministic price process}

One of the key premises underlying the sensitivity analysis that follows is the (exponential) stability of the attractors (price equilibria) to be studied. Thus, at the outset, we need to identify the deterministic map associated with the stochastic law of motion (2), identify its equilibria and discuss their stability. 
If Assumptions 1-2 hold and $\varepsilon_{\alpha}=\varepsilon_{\beta}=\varepsilon_{\bullet}=0$, then (2) takes the form

$$
f(p)= \begin{cases}f_{1}(p)=(1-\alpha+\beta) p+(\alpha-\beta) v+\left(\alpha_{0}-\alpha\right) \gamma, & 0 \leq p<v-\gamma \\ f_{2}(p)=\left(1-\alpha_{0}+\beta\right) p+\left(\alpha_{0}-\beta\right) v, & v-\gamma \leq p \leq v-\epsilon^{-} \\ f_{3}(p)=\left(1-\alpha_{0}\right) p+\alpha_{0} v, & v-\epsilon^{-}<p<v+\epsilon^{-} \\ f_{4}(p)=\left(1-\alpha_{0}+\beta\right) p+\left(\alpha_{0}-\beta\right) v, & v+\epsilon^{-} \leq p \leq v+\gamma \\ f_{5}(p)=(1-\alpha+\beta) p+(\alpha-\beta) v+\left(\alpha-\alpha_{0}\right) \gamma, & v+\gamma<p \leq 1\end{cases}
$$

Key aspects of this piecewise linear map possessing two kinks at $p=v \pm \gamma$ and jump discontinuities at $v \pm \epsilon^{-}$have been analyzed in Jungeilges et al. (2021). Here, we provide a coarse summary of those aspects that are essential for the argument that follows. In particular, the authors focus on a version of the ln-price process that is confined to the unit interval (Jungeilges et al. (2021), Result 4). ${ }^{2}$ After identifying conditions under which $f$ maps the in unit interval $I$ into itself, the authors identify the subset of the parameter space over which three locally stable price equilibria coexist:

$$
p_{1}=v-\frac{\gamma\left(\alpha-\alpha_{0}\right)}{\alpha-\beta}, p_{3}=v, p_{5}=v+\frac{\gamma\left(\alpha-\alpha_{0}\right)}{\alpha-\beta}
$$

The equilibria $p_{1}$ and $p_{5}$ are locally asymptotically stable (1.a.s.) if $\alpha-2<\beta<\alpha-1$, and $p_{3}$, coinciding with the fundamental value, is locally asymptotically stable if $0<\alpha_{0}<2$. In the sequel, we refer to the subset of the 5-dimensional parameter space for which $f: I \rightarrow I$ and $p_{1}$ and $p_{5}$ are l.a.s. as $\Omega^{*}$. For further details and an interpretation of the equilibria from an economic perspective, see Result 5 and the subsequent discussion in Jungeilges et al. (2021).

In the following, we will scrutinize the (i) stochastic sensitivity of these three simple attractors and (ii) study noise-induced transitions that can occur between these attractors. As will become apparent in Sect. 5, our analysis of the transitions between equilibria combines stochastic concepts related to the stochastic sensitivity function to constructs that are central to the study of deterministic maps. For our argument the knowledge of the immediate basins of attraction associated with the equilibria given in (2) is essential. In our case, the immediate basins are given by

$$
\begin{aligned}
& \mathbb{B}\left(p_{1}\right)=\left(b_{1 l}, b_{1 u}\right)=\left(v-\frac{\epsilon^{-}-\left(\alpha-\alpha_{0}\right) \gamma}{1-\alpha+\beta}, v-\epsilon^{-}\right) \\
& \mathbb{B}\left(p_{5}\right)=\left(b_{5 l}, b_{5 u}\right)=\left(v+\epsilon^{-}, v+\frac{\epsilon^{-}-\left(\alpha-\alpha_{0}\right) \gamma}{1-\alpha+\beta}\right) .
\end{aligned}
$$

For a highly constructive account of the concepts critical point and immediate basins, see Sushko et al. (2016) and Avrutin et al. (2019). The cardinality (length) of these basins equals $\left(\alpha-\alpha_{0}\right) \gamma+(\alpha-\beta) v$. Thus, the width depends on within-agent behavioral variation $\alpha-\alpha_{0}$ and between-agents variation in trade intensities when prices

\footnotetext{
2 Confining the natural logarithm of the price to $[0,1]$ corresponds to the requirement $\pi_{t} \in[1, e]$. We can always find a linear transformation that maps observed prices into this interval without affecting, for instance, the underlying autocorrelation structure. Correlation is invariant to linear transformations.
} 


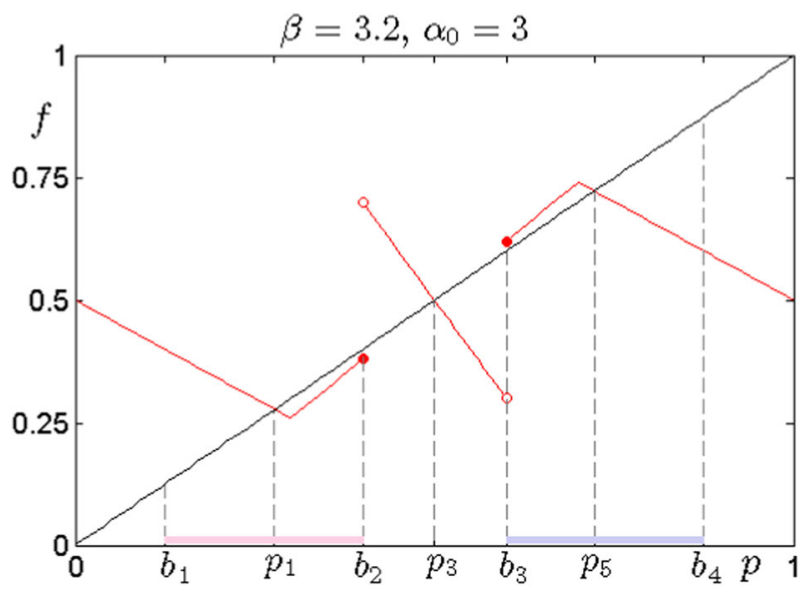

Fig. $1 \quad f(p)$, equilibria and basins $\mathbb{B}\left(p_{1}\right), \mathbb{B}\left(p_{5}\right)$

deviate severely from the fundamental value $v$, i.e., $\alpha-\beta$. Increasing (decreasing) heterogeneity in trading behavior will lead to wider (narrower) immediate basins of attraction. The cardinality of the set of prices mapped into the respective equilibria increases (decreases). A variation in heterogeneity changes the width of the basinsintervals of prices for which the price process converges to equilibria associated with persistent undervaluation $\left(p_{1}\right)$ or overvaluation $\left(p_{5}\right)$-in the same direction.

Figure 1 gives a schematic representation of the graph of the 5-piece map $f(p)$, the equilibria $p_{1}, p_{5}$ with their respective basins $\mathbb{B}\left(p_{1}\right)$ and $\mathbb{B}\left(p_{5}\right)$ superimposed on the $p$-axis. To motivate and/or illustrate our analysis we, will rely on numerical experiments. For fixed values of $\alpha=5, v=0.5, \gamma=0.2, \epsilon^{-}=0.1$, we choose points in $\left(\alpha_{0}, \beta\right)$-space to simulate price paths. Figure 2 shows a partitioning of the set $P=\left\{\left(\alpha_{0}, \beta\right) \mid 0 \leq \alpha_{0} \leq 5 \wedge 0 \leq \beta \leq 4\right\}$. The set of those parameter values for which $f$ does not map the unit interval into itself is indicated by the color gray. The complement of this set is partitioned into three disjoint subsets: (i) the set of $\left(\alpha_{0}, \beta\right)$ values colored blue for which the equilibria $p_{1}$ and $p_{5}$ exist and are stable, (ii) a set for which the equilibria $\left(p_{1}, p_{5}\right)$ exist but are unstable colored in green and (iii) a white set for which attractors other than equilibria exist. Superimposed on this bifurcation diagram are vertical and horizontal broken lines representing parameter constellations for which the price process is simulated in the subsequent sections of the paper. The equilibria associated with the line segments $H=\left\{\left(\alpha_{0}, \beta\right) \mid \alpha_{0}=3 \wedge 3 \leq \beta \leq 4\right\}$ and $V=\left\{\left(\alpha_{0}, \beta\right) \mid 1.7 \leq \alpha_{0} \leq 3.2 \wedge \beta=3.2\right\}$ located in the blue area $\left(p_{1}\right.$ and $p_{5}$ exist and are stable) are plotted against $\beta$ and $\alpha_{0}$, respectively.

In the left subfigure of Fig. $3, p_{3}$ which always exists becomes unstable as $\alpha_{0}$ exceed 2. Under a c.p. variation in $\alpha_{0}$ the distance between $p_{1}$ and $p_{5}$ to $p_{3}$ varies in the opposite direction. A c.p. increase in $\alpha_{0}$ implies that the fundamentalist's trading intensity inside and outside the interval $(v-\gamma, v+\gamma)$ becomes more similar. That is, as the within-heterogeneity goes down the degree of mispricing in long-run states $p_{1}$ and $p_{5}$ decreases. This relationship holds, irrespective of the stability or instability of $p_{3}=v$. 


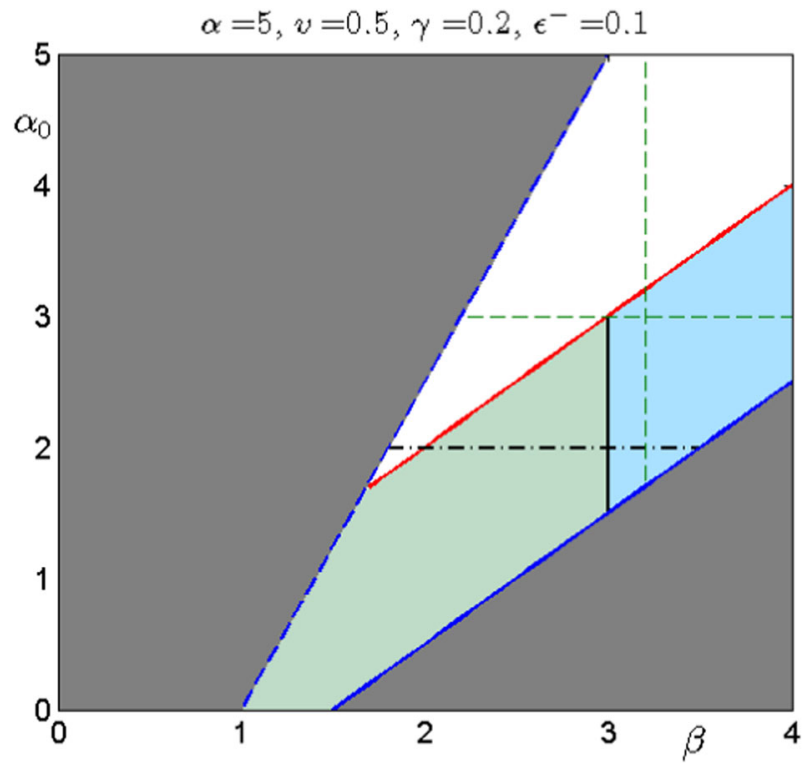

Fig. 2 Bifurcation diagram

The evidence given in the right-hand subfigure of Fig. 3 suggests that a c.p. variation in heterogeneity w.r.t. the trading behavior shown by fundamentalists and chartists when the current asset price does not lie in the vicinity of the fundamental value $p \ni(v-\gamma, v+\gamma)$ triggers a change in the distance of $p_{1}$ and $p_{5}$ from $p_{3}$ in the opposite direction. The intersection of the line segments $H$ and $V(H \cap V)$ gives the parameter constellation considered in the numerical experiments discussed below. At that point in parameter space stable equilibria, $p_{1}$ and $p_{5}$ are coexisting with a unstable $p_{3}$.

\section{Sensitivity analysis via SSF}

The approach to studying the stochastic sensitivity of attractors presented below is due to Mil'shtein and Ryashko (1995). It has been fully developed and popularized by L. Ryashko and his collaborators. In the past, the technique has been successfully applied in the natural sciences to dynamic problems in continuous and discrete time. Only recently has it been utilized to analyze economic processes. The parsimonious presentation that follows gives a rough idea of the concepts. For a more detailed presentation see, for instance Bashkirtseva (2015), Bashkirtseva (2018), and Bashkirtseva and Ryashko (2015).

We can represent the stochastic price process (2) as

$$
p_{t+1}=f\left(p_{t}\right)+\varepsilon g\left(p_{t}\right) \xi_{t}
$$


where $g(\bullet)$ denotes a (not necessarily smooth) function and $\xi_{t}$ signifies an i.i.d Gaussian shock. The following assumption concerning the deterministic skeleton of the stochastic price process (7) is central to the stochastic sensitivity approach.

Assumption 2 For $\varepsilon=0$ (7) has an exponentially stable equilibrium $\bar{p}$.

Let $p_{t}(\varepsilon)$ be the solution of (7) with $p_{0}(\varepsilon)=\bar{p}+\varepsilon v_{0}$, then

$$
z_{t}=\lim _{\varepsilon \rightarrow 0} \frac{p_{t}(\varepsilon)-\bar{p}}{\varepsilon}
$$

characterizes the sensitivity of the price equilibrium $\bar{p}$ to i.i.d. shocks. For the dynamics of $z$, it holds that

$$
z_{t+1}=f^{\prime}(\bar{p}) z_{t}+g(\bar{p}) \xi_{t}
$$

Focussing on the dynamics of second moment $V_{t}=\mathbb{E}\left[z_{t}^{2}\right]$, one can show that

$$
V_{t+1}=\left[f^{\prime}(\bar{p})\right]^{2} V_{t}+g(\bar{p})
$$

holds. It follows from Assumption 2 that $\left|f^{\prime}(\bar{p})\right|<1$. Thus, $V_{t}$ is stabilized for any initial condition. The limit

$$
\omega=\lim _{t \rightarrow \infty} V_{t}=\frac{g^{2}(\bar{p})}{1-\left[f^{\prime}(\bar{p})\right]^{2}}
$$

defines the stochastic sensitivity function (SSF) of the exponentially stable price equilibrium $\bar{p}$. Apparently, an SSF makes sense only if the equilibrium is exponentially stable. In the sequel, we will encounter such a situation in the context of the equilibrium $p_{3}$. If the equilibrium is not stable, then the SSF is irrelevant.

For small noise intensities $\varepsilon$, a stationary distribution of prices exists which is centered at $\bar{p}$. The distribution can be approximated by a normal. In fact, $\varepsilon^{2} \omega$ is related to the variance of the stationary density. Based on the stationary density, it is possible to construct a confidence region around the price equilibrium. The respective $99 \%$ interval takes the form

$$
\bar{p} \pm k \varepsilon \sqrt{2 \omega}
$$

where $k=e r f^{-1}(0.99)$. Thus, the stochastic sensitivity function $\omega$ and the noise intensity $\varepsilon$ determine the boundaries of the confidence interval. 


\section{Sensitivity analysis for the stochastic price process with additive noise}

\subsection{Stochastic sensitivity function for equilibria}

In this case $\varepsilon_{\alpha}=\varepsilon_{\beta}=0, \varepsilon_{\bullet}>0$. With $g(\bar{p})=1$, the stochastic price process is given by

$$
p_{t+1}=f\left(p_{t}\right)+\varepsilon_{\bullet} \xi_{t}
$$

where $f\left(p_{t}\right)$ is given by (3). To obtain the SSFs we need to find the gradient of $f$ :

$$
f^{\prime}(p)= \begin{cases}1-\alpha+\beta, & 0 \leq p<v-\gamma \\ 1-\alpha_{0}+\beta, & v-\gamma \leq p \leq v-\epsilon^{-} \\ 1-\alpha_{0}, & v-\epsilon^{-}<p<v+\epsilon^{-} \\ 1-\alpha_{0}+\beta, & v+\epsilon^{-} \leq p \leq v+\gamma \\ 1-\alpha+\beta, & v+\gamma<p \leq 1\end{cases}
$$

Result 1 Given Assumption 1 holds and $0<\alpha_{0}<2$, then the sensitivity functions for the l.a.s. equilibria $p_{1}, p_{3}, p_{5}$ exist and are given by

$$
\omega_{1}(\alpha, \beta)=\omega_{5}(\alpha, \beta)=\frac{1}{1-(1-\alpha+\beta)^{2}} \text { and } \omega_{3}\left(\alpha_{0}\right)=\frac{1}{1-\left(1-\alpha_{0}\right)^{2}} \text {. }
$$

The condition $\alpha>\beta+1$ ensures that the denominator of $\omega_{1}=\omega_{5}$ is positive. Assuming that $\alpha-2<\beta<\alpha-1$ and $0<\alpha_{0}<2$ hold, the signs of the partial derivatives of the SSF's with respect to the parameters $\alpha, \beta$ and $\alpha_{0}$ are unique:

$$
\frac{\partial \omega_{1}(\alpha, \beta)}{\partial \alpha}>0, \quad \frac{\partial \omega_{1}(\alpha, \beta)}{\partial \beta}<0, \quad \frac{d \omega_{3}\left(\alpha_{0}\right)}{d \alpha_{0}}=\left\{\begin{array}{c}
<0, \alpha_{0}<1 \\
=0, \alpha_{0}=0 \\
>0, \alpha_{0}>1
\end{array}\right.
$$

The stochastic sensitivity of $p_{3}=v$ is determined by the trading response of fundamentalists when they encounter prices in the vicinity of the fundamental value alone. The lowest spread of prices around the fundamental value will be observed for the value of $\alpha_{0}$ which minimizes $\omega_{3}$, i.e., at $\alpha_{0}=1$. At that value the equilibrium $p_{3}$ is super-stable (super-attractive). In that case, prices in the neighborhood of the fundamental values converge to $v$ extremely fast. The spread of the asset price in the neighborhood of $v$ will increase (eventually exponentially) if $\alpha_{0}$ moves toward the end points of the interval $(0,2)$. (The function has poles at 0 and 2.)

The equilibria $p_{1}$ and $p_{5}$ lie in the price intervals $I_{1}=[0, v-\gamma)$ and $I_{5}=$ $(v+\gamma, 1]$, respectively. If the current $p_{t}$ falls into $I_{1}$ or $I_{5}$ then the trading behavior of fundamentalists and chartists is reflected by parameters $\alpha$ and $\beta$. The stochastic sensitivity of the equilibria $p_{1}$ and $p_{5}$ depends only on the respective trade intensities. 


$$
\beta=3.2
$$

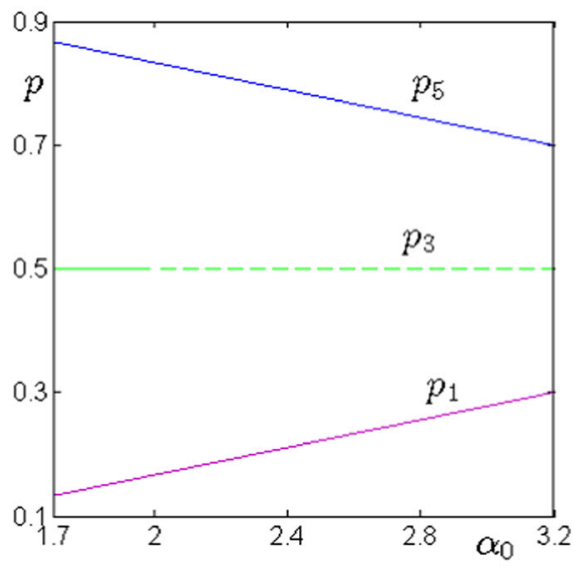

$\alpha_{0}=3$

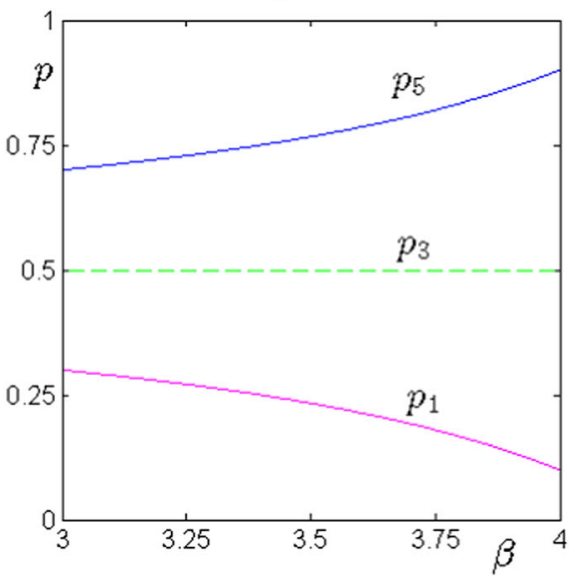

Fig. 3 Comparative statics for $p_{1}, p_{3}, p_{5}$

A marginal c.p. increase in $\beta$ signifies that chartists' trading behavior tends to align with fundamentalists' trading response-as $\alpha$ is fixed and $\alpha>\beta+1$. The partial derivative given above, suggests that the stochastic sensitivity of $p_{1}$ (and $p_{5}$ ) will decrease as a consequence. A decrease in $\beta$ leads to greater heterogeneity and increases the respective SSFs. An interpretation of the marginal c.p. variation in $\alpha$, which according to (15) varies stochastic sensitivity in same direction, renders the same insight. Thus, we can state that if the two agent populations become more homogenous (heterogenous), $p_{1}$ and $p_{5}$ move away from (closer to) the fundamental value (Fig. 3b), but the spread of prices around the respective equilibria tends to decrease (increase).

\subsection{Confidence regions}

As a spin-off from the stochastic sensitivity function technique, we can derive confidence sets for the l.a.s equilibria $p_{1}, p_{3}, p_{5}$. Thus, we let $\mathscr{C}\left(p_{i} ; \varepsilon_{\bullet}\right)=$ $\left(c_{i l}\left(\varepsilon_{\bullet}\right), c_{i u}\left(\varepsilon_{\bullet}\right)\right), i$ in $\{1,3,5\}$, denote the confidence set (interval) associated with equilibrium $p_{i}$ and the noise intensity $\varepsilon_{\bullet}>0$. Our confidence sets are based on (12) and the $3 \sigma$-rule. If the price process involving additive noise is started in a small neighborhood of the equilibrium $p_{i}$, then in approximately $99.72 \%$ of all times the trajectory will stay inside the set $\mathscr{C}\left(p_{i} ; \varepsilon_{\bullet}\right)$. As the cardinality of the confidence sets will play an essential role in our analysis of the transition phenomenon, we will define it next: $\sharp\left[\mathscr{C}\left(p_{i} ; \varepsilon_{\bullet}\right)\right]=L_{i}\left(\varepsilon_{\bullet}\right)=c_{i u}\left(\varepsilon_{\bullet}\right)-c_{i l}\left(\varepsilon_{\bullet}\right)$. Thus, $L_{i}\left(\varepsilon_{\bullet}\right)$ gives the length of the confidence interval centered at $p_{i}, i \in\{1,3,5\}$, given the noise intensity $\varepsilon_{\bullet}$.

Result 2 For $\varepsilon_{\alpha}=\varepsilon_{\beta}=0$ and $\varepsilon_{\bullet}>0$ and given Assumption 1 holds, the confidence sets $\mathscr{C}\left(p_{i} ; \varepsilon_{\bullet}\right), i \in\{1,3,5\}$, for the l.a.s. equilibria $p_{i}$ are given by

$$
c_{1(l, u)}\left(\varepsilon_{\bullet}\right)=v-\frac{\gamma\left(\alpha-\alpha_{0}\right)}{\alpha-\beta} \pm 3 \varepsilon_{\bullet}\left[1-(1-\alpha+\beta)^{2}\right]^{-\frac{1}{2}}
$$




$$
\begin{aligned}
& c_{3(l, u)}\left(\varepsilon_{\bullet}\right)=v \pm 3 \varepsilon_{\bullet}\left[1-\left(1-\alpha_{0}\right)^{2}\right]^{-\frac{1}{2}} \\
& c_{5(l, u)}\left(\varepsilon_{\bullet}\right)=v+\frac{\gamma\left(\alpha-\alpha_{0}\right)}{\alpha-\beta} \pm 3 \varepsilon_{\bullet}\left[1-(1-\alpha+\beta)^{2}\right]^{-\frac{1}{2}}
\end{aligned}
$$

The cardinalities of the resulting confidence sets are given in the following result.

\section{Result 3}

$$
\begin{aligned}
L_{1}\left(\alpha, \beta, \varepsilon_{\bullet}\right) & =L_{5}\left(\alpha, \beta, \varepsilon_{\bullet}\right)=6 \varepsilon_{\bullet}\left[1-(1-\alpha+\beta)^{2}\right]^{-\frac{1}{2}} \\
L_{3}\left(\alpha_{0}, \varepsilon_{\bullet}\right) & =6 \varepsilon_{\bullet}\left[1-\left(1-\alpha_{0}\right)^{2}\right]^{-\frac{1}{2}}
\end{aligned}
$$

The cardinality of confidence set is an increasing function of the noise intensity $\varepsilon_{\bullet}$. The problem of determining how the length $L_{i}\left(\varepsilon_{\bullet}\right)$ of the confidence interval for equilibrium $p_{i}$ is responding to marginal c.p. changes in the trade intensities $\alpha, \alpha_{0}$ and $\beta$ is readily solved. Since $L_{i}\left(\varepsilon_{\bullet}\right)$ constitutes a monotonically increasing transformation of the respective sensitivity function $\omega_{i}$, the signs of the partial derivatives of $L_{i}\left(\varepsilon_{\bullet}\right)$ are identical to those listed in (15):

$$
\frac{\partial L_{1}\left(\alpha, \beta, \varepsilon_{\bullet}\right)}{\partial \alpha}>0 \frac{\partial L_{1}\left(\alpha, \beta, \varepsilon_{\bullet}\right)}{\partial \beta}<0 \frac{d L_{3}\left(\alpha_{0} ; \varepsilon_{\bullet}\right)}{d \alpha_{0}}=\left\{\begin{array}{c}
>0, \alpha_{0}>1 \\
=0, \alpha_{0}=1 \\
<0, \alpha_{0}<1
\end{array}\right\}
$$

The signs are formally correct, but it may be constructive to see whether we can retrace the deep reasons for those signs. If $\alpha$ increases, then the slope of segment $f_{1}(\beta+1-\alpha)$ will be smaller (the slope is negative), so $f_{1}$ becomes steeper. For the deterministic motion around $p_{1}$ that means that the range of iterates increases compared to the baseline $f_{1}$. If noise is added, then this tendency does not vanish. On the other hand, a marginal increase (decrease) in $\beta$ flattens out the slope of $f_{1}$. The range of the iterates (started at $p_{0}$ ) will become narrower. Adding noise does not change this tendency. The length of the confidence interval around $p_{1}$ should decrease.

The same applies in the case of $p_{3}$. For instance, when $\alpha_{0}$ increases beyond 1 , the negative slope of $f_{3}$ becomes steeper which implies a larger range of iterates as the process converges to the fundamental value, than the range implied by the baseline situation. Again, introducing additive noise, does not change the situation.

\subsection{Transition between coexisting equilibria}

The two key constructs in our explanation of transitions occurring between the l.a.s. equilibria $p_{1}$ and $p_{5}$ are the immediate basins $\mathbb{B}\left(p_{i}\right)$ and the confidence intervals $\mathscr{C}\left(p_{i} ; \varepsilon_{\bullet}\right)$ associated with each equilibrium. While the former emerges from the analysis of the deterministic map $f(p)$ in Sect. 3, the latter is a by-product of the stochastic sensitivity function approach applied to the stochastic price law (2). Both sets being anchored at the equilibrium $p_{i}$, they, differ in several aspects. We will argue below 
that a lack of congruence between the sets causes the transitions occurring between coexisting stable equilibria.

A sufficient condition for the existence of transitions between two equilibria can be formulated relying on the binary relation prevailing between these sets. To see this, consider the following cases:

Case $1: \mathscr{C}\left(p_{i} ; \varepsilon_{\bullet}\right) \subset \mathbb{B}\left(p_{i}\right)$

Here, $\mathscr{C}\left(p_{i} ; \varepsilon_{\bullet}\right) \cap \mathbb{B}\left(p_{1}\right)=\mathscr{C}\left(p_{i} ; \varepsilon_{\bullet}\right)$ that is, the prices prevailing in the neighborhood of $p_{i}$ most of the time, are prices which are expected to be mapped into the direction of the equilibrium $p_{i}$. (The "deterministic skeleton" is result of applying expected value operator to 2.) In this case, a price trajectory started in the equilibrium $p_{i}$ will stay in a neighborhood of the equilibrium ad infinitum with given fiducial probability. Such a case is demonstrated in the left subfigure of Fig. 4.

Case $2: \mathscr{C}\left(p_{i} ; \varepsilon_{\bullet}\right) \supset \mathbb{B}\left(p_{i}\right)$

Here the set difference $\mathscr{C}\left(p_{i} ; \varepsilon_{\bullet}\right) / \mathbb{B}\left(p_{i}\right)$ is not empty. There are price states which are likely to occur and which are not going to be mapped in the direction of the equilibrium $p_{i}$. Thus, the price trajectory "escapes" the respective immediate basin and a transition to another equilibrium becomes a likely event. The dynamics evolving in such a case is illustrated in the right-hand subfigure of Fig. 4.

As indicated in Result 3, $L_{i}$ is an increasing function of $\varepsilon_{\bullet}$. Thus, fixing all other parameters, the length of $\mathscr{C}\left(p_{i} ; \varepsilon_{\bullet}\right)$ can be varied independently by choosing alternative noise intensities $0<\varepsilon_{\bullet}<\varepsilon_{\bullet}^{\max }$ where the upper bound is motivated by (i) the endeavor to avoid economically meaningless trajectories and by (ii) the fact that the theory underlying the SSF assumes small noise throughout. A given "case 1," might be transformed into the second case described above by increasing the noise intensity. To provide an illustration of the dynamics resulting from the two cases outlined above, the following parameter constellation was chosen:

$$
\left(\alpha, \alpha_{0}, \gamma, \beta, \epsilon^{-}\right)=(5,3,0.2 ; 3.2,0.1)
$$

It implies that the coexisting equilibria $p_{1}$ and $p_{5}$ are l.a.s, while $p_{3}=v=0.5$ is unstable. Figure 4 depicts the stochastic dynamics as it unfolds in the state space (upper panels) as well as in the time domain (lower panels) for two different levels of noise $\varepsilon_{\bullet}=0.01$ (left column) and $\varepsilon_{\bullet}=0.04$ (right column).

Both constructs, confidence regions (yellow) and immediate basins $\left(\mathbb{B}\left(p_{1}\right)\right.$ pink and $\mathbb{B}\left(p_{5}\right)$-blue $)$ are superimposed on the horizontal axis in the state space representation. The simulated price series are shown in the form of an iterative Lamerey diagram. If the series starts in $p_{1}\left(p_{5}\right)$ its elements are colored pink (blue). In each subfigure, the lower panel shows a time-domain representation of the processes. The sample series are superimposed on the respective basins (pink and blue) and confidence regions (yellow).

In the small noise scenario $\left(\varepsilon_{\bullet}=0.01\right)$, the confidence set is contained in the respective immediate basin. At such a low noise level, an escape from the basin is a highly unlikely event. Our time domain representation shows the motion of both price series within the confidence bands $\mathbb{C}\left(p_{1} ; 0.01\right)$ and $\mathbb{C}\left(p_{5} ; 0.01\right)$, which are embedded in the respective basins $\mathbb{B}\left(p_{1}\right)$ and $\mathbb{B}\left(p_{5}\right)$, throughout the entire observation period. 

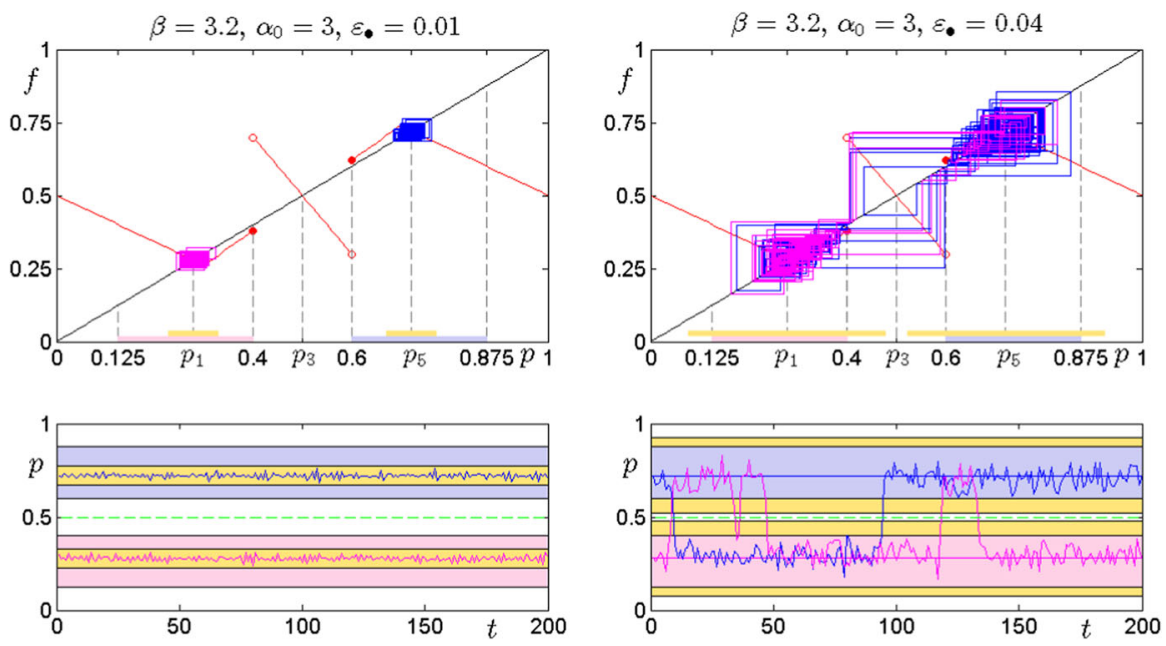

Fig. 4 Stochastic states

The situation changes drastically, once the noise intensity is increased to $\varepsilon_{\bullet}=0.04$. As shown in the right subfigure, the cardinality of the confidence sets increase such that the respective basins are now subsets of the confidence sets. There are highly probable states (prices) which would not be mapped into $p_{1}$ or $p_{5}$ under the deterministic system. An escape from a basin is highly likely in this scenario. The lower panel shows how the price process starts close to $p_{5}$-initially the asset is over-valued, undergoes a transition after a few iterations, stays close to the low level equilibrium for an extended period, and returns to the neighborhood of $p_{5}$ after some iterations. Similar, recurrent transitions between equilibria are observed when the price process (sample series colored pink) starts in the low level equilibrium $p_{1}$. As in the previous case, the transitions occurring here are merely induced by an increase in the variance of the noise signal reflecting a higher degree of uncertainty in the market environment.

Evidently, the numerical example reveals two types of transitions between the l.a.s equilibria $p_{1}$ and $p_{5}: p_{1} \rightarrow p_{5}$ and $p_{5} \rightarrow p_{1}$. In principle, each type of transition can occur in two ways: the price trajectory ends up in the interval $\left[c_{i l}\left(\varepsilon_{\bullet}\right), b_{i l}\right]$ (escape via the lower bound of $\mathbb{B}\left(p_{i}\right)$ ) or in $\left[b_{i u}, c_{i u}\left(\varepsilon_{\bullet}\right)\right]$ (escape via the upper bound of $\mathbb{B}\left(p_{i}\right)$. Under the parameter constellation at hand, we find that the escape from $\mathbb{B}\left(p_{1}\right)$ can be expected to occur via the upper bound $b_{1 u}$, while the escape from $\mathbb{B}\left(p_{2}\right)$ tends to emerge via the lower bound $b_{2 l}$.

\subsection{Critical intensities}

Central to our discussion below is the concept of critical intensity, that is, the largest noise level for which transitions are still unlikely events. To frame it alternatively: If this critical noise level is exceeded, then transitions become observable events. 
Definition 4 The noise level for which $b_{i e}=c_{i e}\left(\varepsilon_{\bullet}\right), e \in\{l, u\}$, holds is referred to as the critical noise intensity for escaping $\mathbb{B}\left(p_{i}\right)$ via the boundary $b_{i e}$. It is denoted as $\varepsilon_{\bullet i, e}^{*}$.

Since $c_{i l}\left(\varepsilon_{\bullet}\right)$ and $c_{i u}\left(\varepsilon_{\bullet}\right)$, apart from being (i) monotonically decreasing/increasing in $\varepsilon_{\bullet}$, the bounds are invertible w.r.t. $\varepsilon_{\bullet}$. In our case, we can obtain a closed form of an analytical solution for the critical noise intensities. For other examples of analytically determined critical intensities, see for instance Jungeilges et al. (2018), Bashkirtseva and Ryashko (2017), and Bashkirtseva et al. (2016).

Result 4 Given Assumption 1 holds and $p_{1}, p_{5}$ are locally asymptotically stable, then critical intensities exist and are equal to

$$
\begin{aligned}
& \varepsilon_{\bullet 1, l}^{*}=\varepsilon_{\bullet 5, u}^{*}=\frac{1}{3} \frac{\epsilon^{-}(\alpha-\beta)-\gamma\left(\alpha-\alpha_{0}\right)}{(1-\alpha+\beta) \sqrt{\alpha-\beta}} \sqrt{2-\alpha+\beta} \\
& \varepsilon_{\bullet 1, u}^{*}=\varepsilon_{\bullet 5, l}^{*}=\frac{1}{3} \frac{\gamma\left(\alpha-\alpha_{0}\right)-\epsilon^{-}(\alpha-\beta)}{\sqrt{\alpha-\beta}} \sqrt{2-\alpha+\beta} .
\end{aligned}
$$

From a formal point of view, critical intensities are functions $\varepsilon_{\bullet i, e}^{*}: \Omega^{*} \rightarrow \mathbb{R}_{+}$ which map points in the restricted parameter space $\Omega^{*}$ onto the positive real line (noise intensities). In our context, Assumption 1 implies that in (23) and (24) the denominator is a positive real number, while the stability of $p_{i}(\beta+1<\alpha<\beta+2)$ ensures that $\sqrt{2-\alpha+\beta} \in \mathbb{R}_{+}$.

The critical intensities depend in a complex way on all parameters. Note how the threshold prices $\epsilon^{-}$and $\gamma$ are concentrated in the numerators of (23) and (24) critical intensities. Even though the aspect of threshold prices is not the focus of this paper, we should point out that the changes in the difference between $\gamma$ and $\epsilon^{-}$will have an effect on the critical intensities.

To illustrate the concept of critical intensities and to introduce an alternative interpretation of it, we rely on evidence from simulations based on the parameter constellation given in (22). In Fig. 5, the vertical axis of the graph is associated with the state space $I$ of the stochastic price process (2) while the horizontal axis holds a fine grid $(\Delta=0.0005)$ noise intensities from the interval $(0,0.05)$. For given level of $\varepsilon_{\bullet}$, we fix an initial price in a small neighborhood of the equilibrium $p_{1}$ and generate a single stochastic price trajectory of length $T=200$. The realizations are plotted against the respective level of $\varepsilon_{\bullet}$. In addition, the basin $\mathbb{B}\left(p_{1}\right)$ which is independent of $\varepsilon_{\bullet}$ and the confidence region $\mathbb{C}\left(p_{1} ; \varepsilon_{\bullet}\right)$ (end points indicated by broken lines) are superimposed on the graph.

As the noise intensities increase, the spread of the asset prices around $p_{1}$ increases, but the bulk of the realizations are elements of $\mathbb{B}\left(p_{1}\right)$ as the confidence regions $\mathbb{C}\left(p_{1} ; \varepsilon_{\bullet}\right)$ are true subsets of the basin. At $\varepsilon_{\bullet 1, u}^{*} \approx 0.02445$ the situation changes. The basin becomes a subset of the confidence regions. Prices tend to "escape" $\mathbb{B}\left(p_{1}\right)$ via the boundary $b_{1 u}$ and tend to be mapped into the vicinity of $p_{5}$. A transition between two l.a.s. equilibria ( $p_{1} \rightarrow p_{5}$ ) occurs. Eventually, the process leaves the basin of the equilibrium in which the asset is over-priced $\left(p_{5}\right)$ to return to states in which the asset is under-valued $\left(p_{1}\right)\left(p_{1} \leftarrow p_{5}\right)$. Under increasing levels of noise, 
Fig. 5 Stochastic trajectories versus levels of $\varepsilon_{\bullet}$

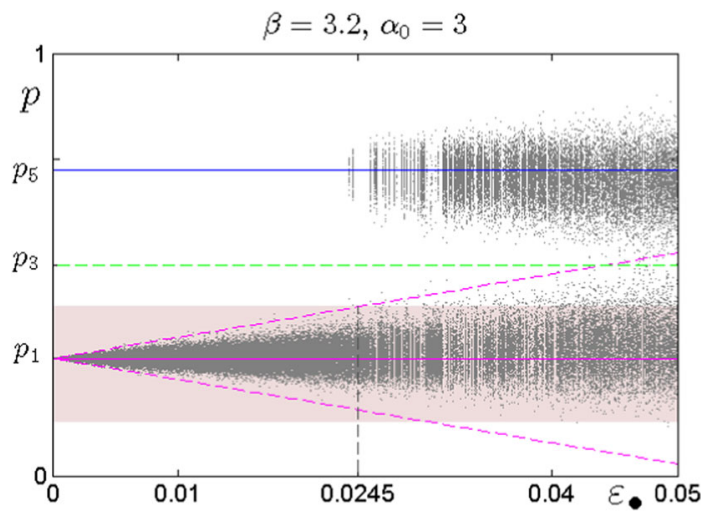

Fig. 6 Marginal densities for alternative values of $\varepsilon_{\bullet}$

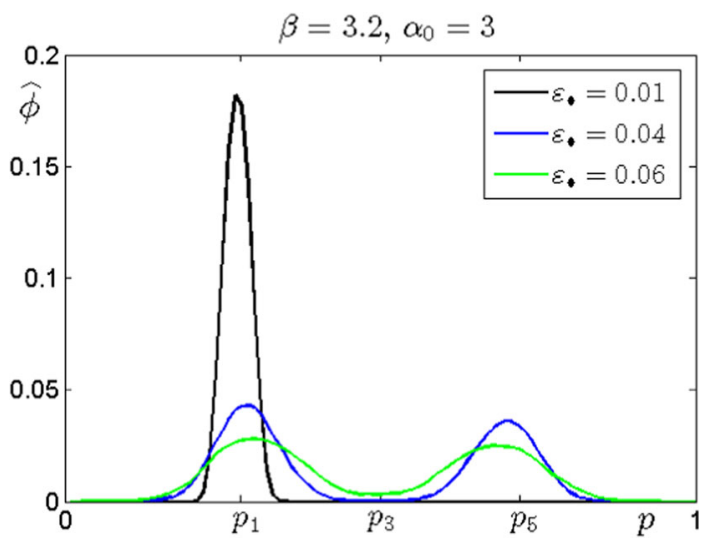

recurrent transitions - in both directions-prevail. Although the graph in Fig. 5 allows for a first look at the properties of the price distribution for each $\varepsilon_{\bullet}$ and effectively reveals the evolution from a uni-modal to a bi-modal density of prices as the noise intensity increases, we provide a more detailed account of the distribution of prices in Fig. 6.

For each level of $\varepsilon_{\bullet}$ there exists a density function $\phi_{\varepsilon_{\bullet}}(p)$ describing the distribution of prices on the unit interval. In Fig. 6, we exhibit the smoothed-histogram estimates $\hat{\phi}_{\varepsilon_{\bullet}}(p)$ for selected noise intensities $\varepsilon_{\bullet} \in\{0.01,0.04,0.06\}$ based on single simulation runs. Apparently, the uni-modal density centered at $p_{1}$ evolves into a bi-modal one under increasing uncertainty. Apparently, the bi-modal marginal densities associated with $\varepsilon_{\bullet}=0.04$ and $\varepsilon_{\bullet}=0.06$ posses a local minimum at the fundamental value $p_{3}=v$. This minimum is invariant to changes in the noise level. Recently, Schmitt and Westerhoff (2017) have provided empirical evidence for bi-modality in the S\&P500. Moreover, the authors establish that this property of asset prices can be generated in the context of several agent-based asset market models.

In fact, in the family (space) of densities $\phi_{\varepsilon_{\bullet}}(p)$, a bifurcation occurs for some level of $\varepsilon_{\bullet} \in(0,0.05)$. The critical intensities given in Result 4 can be used to approximate 
noise intensity at which such a bifurcation occurs. In the case at hand, the bifurcation materializes approximately at the noise intensity of $0.02 \overline{4}$.

So far, we have focussed on additive noise alone. In the following section, we will consider the variants of our stochastic model in which the trading intensities of either fundamentalists (case 2) or chartists (case 3) are subject to random shocks. After summarizing our results from the respective analyses, we compare the sensitivity of the coexisting equilibria $p_{1}$ and $p_{5}$ under different types of noise.

\section{Parametric noise}

\subsection{Parametric noise affecting $\alpha$}

In the case $\varepsilon_{\alpha}>0, \varepsilon_{\beta}=0, \varepsilon_{\bullet}=0$, only the segments $f_{1}$ and $f_{5}$ of the map $f(p)$ are affected by parametric shocks. Thus, (2) can be represented as

$$
p_{t+1}=f\left(p_{t}\right)+\varepsilon g\left(p_{t}\right) \xi_{t}
$$

where

$$
g_{\alpha}(p)= \begin{cases}v-\gamma-p, & 0 \leq p<v-\gamma \\ 0, & v-\gamma \leq p \leq v-\epsilon^{-} \\ 0, & v-\epsilon^{-}<p<v+\epsilon^{-} \\ 0, & v+\epsilon^{-} \leq p \leq v+\gamma \\ v+\gamma-p, & v+\gamma<p \leq 1\end{cases}
$$

By implementing the approach described in Sect. 4, we obtain the stochastic sensitivity functions for the l.a.s. equilibria, determine the associated confidence regions and finally calculate the respective critical intensities. Our findings are summarized in the sequence of results given below. Under the parameter constraints implied by Assumption 1 and the stability conditions already outlined in the case of additive noise, all constructs exist and are well-defined.

Result 5 If $\varepsilon_{\alpha}>0, \varepsilon_{\beta}=0, \varepsilon_{\bullet}=0$, then the sensitivity functions for the equilibria $p_{1}, p_{3}, p_{5}$ based on (11) equal

$$
w_{\alpha}\left(p_{1}\right)=w_{\alpha}\left(p_{5}\right)=\frac{\gamma^{2}\left(\beta-\alpha_{0}\right)^{2}}{(\alpha-\beta)^{3}(2-\alpha+\beta)} \text { and } w_{\alpha}\left(p_{3}\right)=0 \text {. }
$$

Since the center segment $f_{3}$ of $f(p)$ is not affected by noise, it is deterministic. We define the sensitivity for such cases to be 0 . Since the comparative perspective adopted in the sequel of this section will focus the stable equilibria $p_{1}$ and $p_{5}$, we will consider confidence sets and critical intensities for these equilibria only. 
Result 6 Given $\varepsilon_{\alpha}>0, \varepsilon_{\beta}=0, \varepsilon_{\bullet}=0$, the confidence sets $\mathscr{C}\left(p_{i} ; \varepsilon_{\alpha}\right), i \in\{1,3,5\}$, are given by

$$
\begin{aligned}
& c_{1(l, u)}\left(\varepsilon_{\alpha}\right)=v-\frac{\gamma\left(\alpha-\alpha_{0}\right)}{\alpha-\beta} \pm 3 \varepsilon \alpha \sqrt{\frac{\gamma^{2}\left(\beta-\alpha_{0}\right)^{2}}{(\alpha-\beta)^{3}(2-\alpha+\beta)}} \\
& c_{5(l, u)}\left(\varepsilon_{\alpha}\right)=v+\frac{\gamma\left(\alpha-\alpha_{0}\right)}{\alpha-\beta} \pm 3 \varepsilon_{\alpha} \sqrt{\frac{\gamma^{2}\left(\beta-\alpha_{0}\right)^{2}}{(\alpha-\beta)^{3}(2-\alpha+\beta)}} .
\end{aligned}
$$

Finally, we present our findings concerning the critical intensities for the case in which the trade intensities of the fundamentalists are prone to shocks.

Result 7 In the case of $\varepsilon_{\alpha}>0, \varepsilon_{\beta}=0, \varepsilon_{\bullet}=0$, the critical intensities are given by

$$
\begin{aligned}
& \varepsilon_{\alpha 1, l}^{*}=\varepsilon_{\alpha 5, u}^{*}=\frac{1}{3} \frac{\epsilon^{-}(\alpha-\beta)-\gamma\left(\alpha-\alpha_{0}\right)}{\gamma(1-\alpha+\beta)\left(\beta-\alpha_{0}\right)} \sqrt{(2-\alpha+\beta)(\alpha-\beta)} \\
& \varepsilon_{\alpha 1, u}^{*}=\varepsilon_{\alpha 5, l}^{*}=\frac{1}{3} \frac{\gamma\left(\alpha-\alpha_{0}\right)-\epsilon^{-}(\alpha-\beta)}{\gamma\left(\beta-\alpha_{0}\right)} \sqrt{(2-\alpha+\beta)(\alpha-\beta)} .
\end{aligned}
$$

A detailed discussion about how specific parameter changes affect the construct is, of course, possible but will not be included here to honor the scope of this paper. Instead, we will summarize our findings for the case in which noise affects the parameter $\beta$.

\subsection{Parametric noise affecting $\beta$}

In the case $\varepsilon_{\alpha}=0, \varepsilon_{\beta}>0, \varepsilon_{\bullet}=0$, the segments $f_{1}, f_{2}, f_{4}$, and $f_{5}$ of the map $f(p)$ are affected by the specific parametric shock. Thus, (2) can be represented as

$$
p_{t+1}=f\left(p_{t}\right)+\varepsilon g\left(p_{t}\right) \xi_{t}
$$

where

$$
g_{\beta}(p)=\left\{\begin{array}{l}
v-p, 0 \leq p<v-\gamma \\
v-p, v-\gamma \leq p \leq v-\epsilon^{-} \\
0, \quad v-\epsilon^{-}<p<v+\epsilon^{-} \\
v-p, v+\epsilon^{-} \leq p \leq v+\gamma \\
v-p, v+\gamma<p \leq 1
\end{array}\right.
$$

As in the context of $\alpha$-noise, we state that all functions given in this summary of results exist and are well-defined when we consider the restricted parameter space $\Omega^{*}$ and invoke Assumption 1.

Result 8 If $\varepsilon_{\alpha}=0, \varepsilon_{\beta}>0, \varepsilon_{\bullet}=0$, then the sensitivity functions for the equilibria $p_{1}, p_{3}, p_{5}$ based on (11) equal

$$
w_{\alpha}\left(p_{1}\right)=w_{\alpha}\left(p_{5}\right)=\frac{\gamma^{2}\left(\alpha-\alpha_{0}\right)^{2}}{(\alpha-\beta)^{3}(2-\alpha+\beta)} \text { and } w_{\alpha}\left(p_{3}\right)=0 .
$$


Again, we only consider the confidence regions for the equilibria $p_{1}$ and $p_{5}$ since our comparative perspective will focus on those coexisting equilibria.

Result 9 Given $\varepsilon_{\alpha}=0, \varepsilon_{\beta}>0, \varepsilon_{\bullet}=0$, the confidence sets $\mathscr{C}\left(p_{i} ; \varepsilon_{\beta}\right), i \in\{1,3,5\}$, are given by

$$
\begin{aligned}
& c_{1(l, u)}\left(\varepsilon_{\beta}\right)=v-\frac{\gamma\left(\alpha-\alpha_{0}\right)}{\alpha-\beta} \pm 3 \varepsilon \beta \sqrt{\frac{\gamma^{2}\left(\alpha-\alpha_{0}\right)^{2}}{(\alpha-\beta)^{3}(2-\alpha+\beta)}} \\
& c_{5(l, u)}\left(\varepsilon_{\beta}\right)=v+\frac{\gamma\left(\alpha-\alpha_{0}\right)}{\alpha-\beta} \pm 3 \varepsilon_{\beta} \sqrt{\frac{\gamma^{2}\left(\alpha-\alpha_{0}\right)^{2}}{(\alpha-\beta)^{3}(2-\alpha+\beta)}} .
\end{aligned}
$$

As in the previously scrutinized cases, also in the case of $\beta$-noise, closed-form analytical solutions can be given for the critical intensities given in Definition 4 .

Result 10 In the case of $\varepsilon_{\alpha}=0, \varepsilon_{\beta}>0, \varepsilon_{\bullet}=0$, the critical intensities are given by

$$
\begin{aligned}
& \varepsilon_{\beta 1, l}^{*}=\varepsilon_{\beta 5, u}^{*}=\frac{1}{3} \frac{\epsilon^{-}(\alpha-\beta)-\gamma\left(\alpha-\alpha_{0}\right)}{(1-\alpha+\beta) \gamma\left(\alpha-\alpha_{0}\right)} \sqrt{(2-\alpha+\beta)(\alpha-\beta)} \\
& \varepsilon_{\beta 1, u}^{*}=\varepsilon_{\beta 5, l}^{*}=\frac{1}{3} \frac{\gamma\left(\alpha-\alpha_{0}\right)-\epsilon^{-}(\alpha-\beta)}{\gamma\left(\alpha-\alpha_{0}\right)} \sqrt{(2-\alpha+\beta)(\alpha-\beta)}
\end{aligned}
$$

The results outlined above, will be used to compare the sensitivity of the coexisting attractors $p_{1}$ and $p_{5}$ under different noise scenarios.

\subsection{Comparative perspective}

For the parameter constellations chosen for the numerical experiments of the preceding sections, we will contrast the sensitivity functions and critical intensities for the equilibria $p_{1}$ and $p_{5}$ across the three types of noise. Recall that for the $\alpha_{0}$ used in the experiments the equilibrium $p_{3}=v=0.5$ is unstable.

Figure 7 clearly provides evidence for the fact that the sensitivity function $\omega_{1}=\omega_{5}$ for additive noise clearly dominates the sensitivity functions for the parametric noise scenarios. In a sense, an additive shock affects the entire graph of $f(p)$ while in the case of $\beta$-noise the slopes of $f_{1}, f_{2}, f_{3}, f_{4}$ are random while the center segment stays deterministic. Under $\alpha$-noise, only the slopes of $f_{1}$ and $f_{5}$ are random, while everything else stays deterministic. The experiment suggests that stochastic sensitivity of the stable equilibria increases in the number of segments affected by shocks.

Seen from an economic perspective this results seems to be in line with intuition. The noise affecting the price formation process as a whole (affecting the process in which the demand signals are transformed into price movements) should be expected to render larger price movements around 1.a.s. equilibria than shocks that affect the trading intensities of fundamentalists or chartists alone.

The left panel of Fig. 8 exhibits critical intensities for scenarios in which $\beta$ is fixed at 3.2 while $\alpha_{0}$ varies between 1.7 and 3.2. Under additive noise, we will observe 


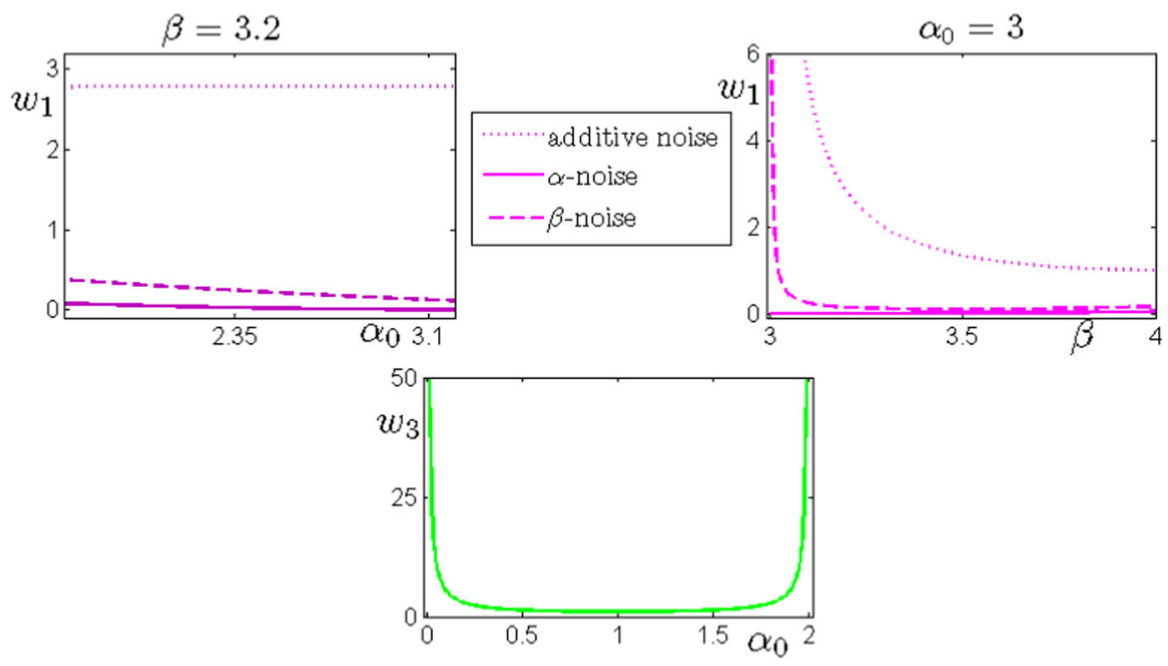

Fig. 7 Comparison of stochastic sensitivity functions
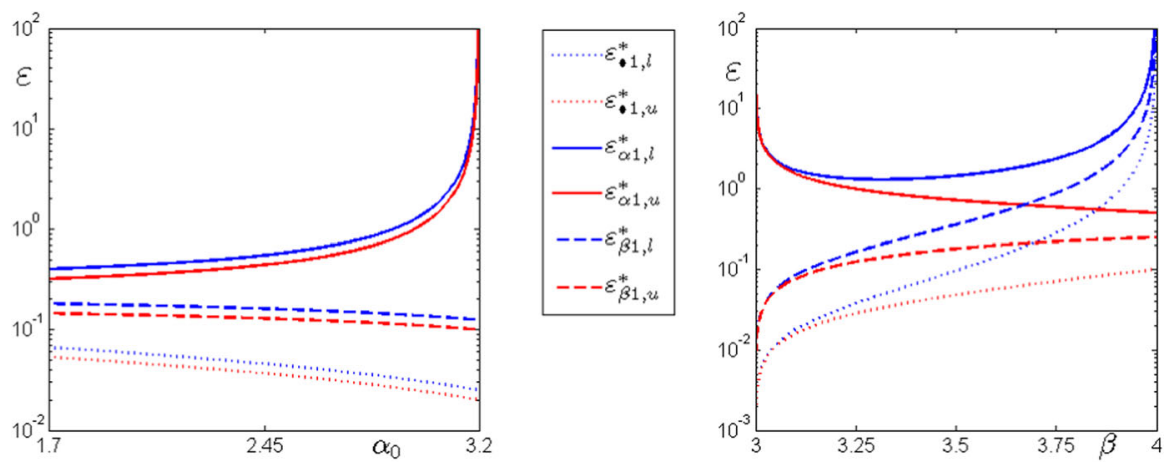

Fig. 8 Comparison of critical intensities (log-scale)

transitions of the types $p_{1} \rightarrow p_{5}$ and $p_{5} \rightarrow p_{1}$ for comparatively low noise intensities. The price process tends to escape the basin $\mathbb{B}\left(p_{1}\right)$ via the upper endpoint already at lower levels of noise irrespective of the noise considered. The motion around the l.a.s. equilibria will be much more robust to changes in the noise intensity if only the trade intensities of specific groups of agents are prone to shocks. Also note how an increase in $\alpha_{0}$ lowers the critical sensitivity in the case of additive- and $\beta$-noise, but increases it for $\alpha$-noise. Thus, transitions from one stable attractor to another become unlikely as $\alpha_{0}$ approaches 3.2.

The right panel of Fig. 8 shows critical intensities for the three types of noise presented on a log-scale for the situation in which $\alpha_{0}=3$ is fixed and $\beta$ varies between 3 and 4 .

Under additive noise, the critical intensities for leaving $\mathbb{B}\left(p_{1}\right)$ increase as the chartists, on average, trade more aggressively. The critical intensity for leaving via the upper bound $\varepsilon_{\bullet 1, u}^{*}$ grows less than $\varepsilon_{\bullet 1, l}^{*}$ as $\beta$ increases. Escapes from $\mathbb{B}\left(p_{1}\right)$ via the 
upper boundary of the corresponding basin will be observed at lower noise intensities than escapes via the lower end of the basin. The motion of asset prices around the $p_{1}$ becomes a more robust phenomenon as the chartists, on average, choose to trade more aggressively.

Qualitatively, the case of $\beta$-noise resembles the case of additive noise. The main difference being that the critical intensities under $\beta$-noise are higher for all $\beta$ value considered. Escapes happen only at higher levels of the noise intensity. Motion around a price level at which the asset is miss-priced is a more robust phenomenon than in the case of additive noise.

In the least sensitive case of $\alpha$-noise, $\varepsilon_{\alpha 1, u}^{*}$ decreases as trade intensities of chartists increases. The motion around the equilibrium price becomes a less robust phenomenon as the chartists trade more aggressively.

\section{Discussion and conclusion}

The extant literature on deterministic asset price dynamics referenced in Sect. 1 demonstrates how bull and bear markets can emerge endogenously. They indeed exist across different model specifications and are typically instigated through changes in investor trading behavior. In the preceding sections, we have shown how such bull and bear markets can evolve if the economic environment becomes sufficiently noisy or uncertain. Eventually, the confidence regions around the equilibria are not proper subsets of the respective basins anymore, and a process of recurrent transitions between locally asymptotically stable equilibria starts. The noise levels at which transitions become likely depends on agents' trading intensities in an intricate way. Moreover, we establish that the propensity for observing transitions is the highest under additive noise.

Our model differs from existing models in the tradition of Day and Huang in an important aspect. We allow for differences in the price thresholds $\gamma$ and $\epsilon^{-}$. As demonstrated in Jungeilges et al. (2021) such a disparity leads to phenomena in the context of price equilibria that are not present if $\gamma=\epsilon^{-}$holds. According to Results 4, 7 and 10 , the critical intensities also depend on those thresholds. To clarify in how far they play a role for the onset of transitions will be the subject of another investigation.

In the preceding sections, we concentrated on the variation in (i) noise intensities as well as on different (ii) types of noise. But it is also possible that at a given level of noise, the variation of a parameter, i.e. a change in the behavior of agents might induce transitions between stable equilibria. To substantiate that claim we consider a variation in the fundamentalists' trade intensity in the neighborhood of the unstable fundamental value. Figure 9 illustrates the effects of such a c.p. variation in $\alpha_{0}$. Varying this parameter will change the levels of $p_{1}$ and $p_{5}$. As $\alpha_{0}$ increases, the equilibria move closer to $p_{3}=v$, thus mispricing in the equilibria becomes less pronounced.

Figure 9 shows how important the conditions around the unstable equilibrium are. Note that the noise intensity is fixed. Only $\alpha_{0}$ varies and exceeds the levels for which the fundamental value is locally asymptotically stable. The nature of the transition itself (duration, location) depends on the parameter reflecting trading intensity of the fundamentalists in the neighborhood of the unstable fundamental value. The variation of $\alpha_{0}$ has two effects: (i) the levels of $p_{1}$ and $p_{5}$ change such that the respective 
Fig. 9 A c.p. variation in $\alpha_{0}$ : states, basins and confidence bands

Fig. 10 Densities for alternative $\alpha_{0}$
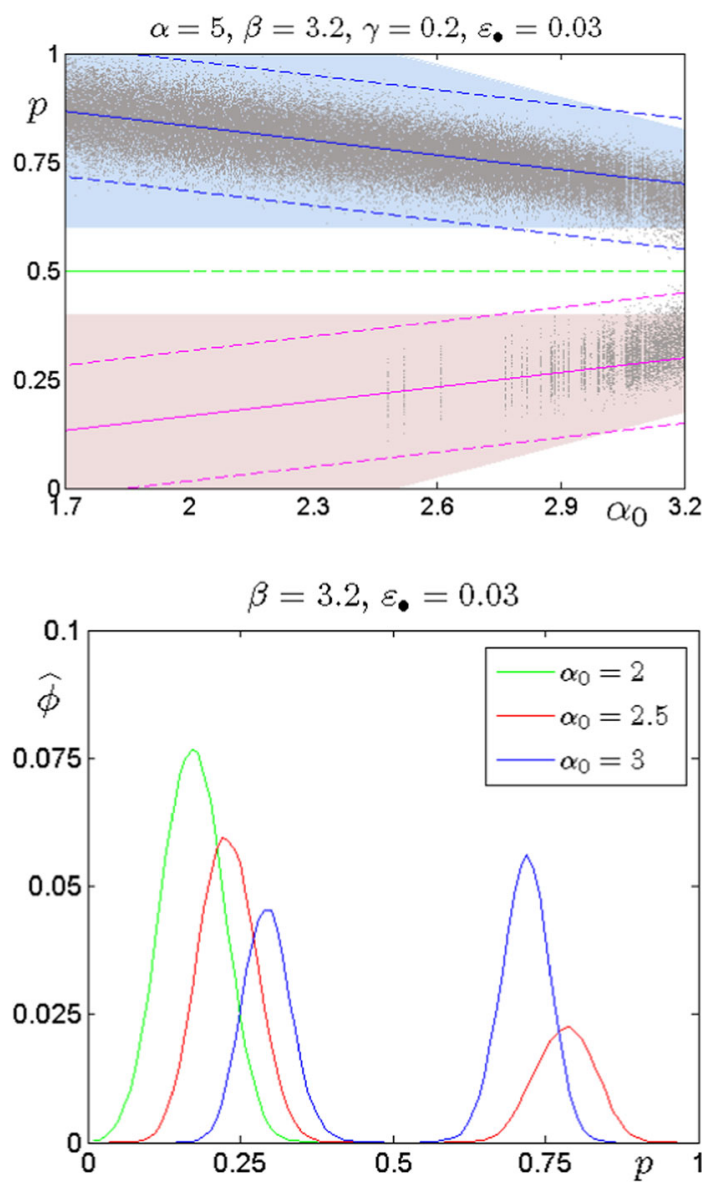

distances to $p_{3}=v$ vary in the opposite direction and (ii) it changes the cardinalities of $\mathbb{B}\left(p_{1}\right)$ and $\mathbb{B}\left(p_{5}\right)$ in the opposite direction. But the length of the confidence intervals (19) is independent of $\alpha_{0}$. Thus, in the case at hand, increasing $\alpha_{0}$ will eventually lead to transitions between the locally stable $p_{1}$ and $p_{5}$, apparently via $b_{1 u}$ and $b_{5 l}$. Figure 10 illustrates the effect of variations in $\alpha_{0}$ on the stationary density of the asset prices. Eventually, a bifurcation from a uni-modal to a bi-modal density occurs.

In particular, at $\alpha_{0}=2$ the asset price trajectory spends more time in the bear market than in the bull market. As the fundamentalists trade more aggressively in the neighborhood of the fundamental value $-\alpha_{0}$ increases to 3 - the resulting bi-modal marginal density suggests that the observed price trajectory spends more time in the bull market than in the bear market. Exactly this quality has been observed for the S\&P500 studied in Schmitt and Westerhoff (2017). While this fact is worthwhile mentioning, we should emphasize that the evidence given in Fig. 10 is based on single simulation runs (one run per parameter value).

The techniques applied, in a sense, allow for a theoretical "stress" test of technically stable states of an asset market. As demonstrated, noise effects and effects of 
behavioral changes on the sensitivity of equilibria can be neatly separated or studied in combination. The relevance of this type of analysis derives from the fact that it might help us to further our understanding of the asset price dynamics in the presence of high environmental volatility.

Future projects will concentrate, first of all, on a systematic analysis and interpretation of the critical intensities found for the cases of parametric noise. Moreover, the price dynamics under a combination of types of noise-possibly correlated-will be studied. One should try to understand how the statistical properties of the price process, for instance its autocorrelation function or selected moments, are related to the parameters of the models, i.e. to investor behavior. Finally, it would be interesting to contrast the statistical properties of the simulated price series with known salient features of real financial times series.

Funding Open Access funding provided by University of Agder.

\section{Compliance with ethical standard}

Conflict of interest The authors declare that they have no conflict of interest.

Open Access This article is licensed under a Creative Commons Attribution 4.0 International License, which permits use, sharing, adaptation, distribution and reproduction in any medium or format, as long as you give appropriate credit to the original author(s) and the source, provide a link to the Creative Commons licence, and indicate if changes were made. The images or other third party material in this article are included in the article's Creative Commons licence, unless indicated otherwise in a credit line to the material. If material is not included in the article's Creative Commons licence and your intended use is not permitted by statutory regulation or exceeds the permitted use, you will need to obtain permission directly from the copyright holder. To view a copy of this licence, visit http://creativecommons.org/licenses/by/4.0/.

\section{References}

Avrutin V, Gardini L, Sushko I, Tramontana F (2019) Continuous and discontinuous piecewise-smooth onedimensional maps World Scientific Series on Nonlinear Science Series A, vol 95. World Scientific, Singapore. https://doi.org/10.1142/8285

Bashkirtseva I (2015) Stochastic phenomena in one-dimensional Rulkov model of neuronal dynamics. Discrete Dyn Nat Soc. https://doi.org/10.1155/2015/495417

Bashkirtseva I (2018) Crises, noise, and tipping in the hassell population model. Chaos 28(3):033603. https://doi.org/10.1063/1.4990007

Bashkirtseva I, Ryashko L (2015) Approximating chaotic attractors by period-three cycles in discrete stochastic systems. Int J Bifurc Chaos 25(10): 15501388. https://doi.org/10.1142/S0218127415501382

Bashkirtseva I, Ryashko L (2017) Stochastic sensitivity analysis of noise-induced order-chaos transitions in discrete-time systems with tangent and crisis bifurcations. Physica A Stat Mech Appl 467:573-584. https://doi.org/10.1016/j.physa.2016.09.048

Bashkirtseva I, Nasyrova V, Ryashko L, Tsvetkov I (2016) Noise-induced intermittency and transition to chaos in the neuron Rulkov model. Vestnik Udmurtskogo Universiteta Matematika Mekhanika Komp'yuternye Nauki 26(4):453-462 10.20537/vm160401

Belyaev A, Ryazanova T (2019a) Mechanisms of spikes generation in piecewise rulkov model. In: Volkovich V, Zvonarev S, Kashin I, Smirnov A, Narkhov E (eds) Physics, technologies and innovation, PTI 2019, American Institute of Physics Inc., United States, AIP Conference Proceedings. https://doi.org/10. 1063/1.5134235

Belyaev A, Ryazanova T (2019b) The stochastic sensitivity function method in analysis of the piecewisesmooth model of population dynamics. Izv IMI UdGU 53:36-47 10.20537/2226-3594-2019-53-04 
Belyaev A, Ryazanova T (2019c) Stochastic sensitivity of attractors for a piecewise smooth neuron model. J Differ Equ Appl 25(9-10):1468-1487. https://doi.org/10.1080/10236198.2019.1678596

Böhm V, Wenzelburger J (2005) On the performance of efficient portfolios. J Econ Dyn Control 29(4):721740. https://doi.org/10.1016/j.jedc.2004.01.006

Brock WA, Hommes CH (1998) Heterogeneous beliefs and routes to chaos in a simple asset pricing model. J Econ Dyn Control 22(8):1235-1274. https://doi.org/10.1016/S0165-1889(98)00011-6

Cafferata A, Tramontana F (2019) A financial market model with confirmation bias. Struct Change Econ Dyn 51:252-259. https://doi.org/10.1016/j.strueco.2019.08.004

Chiarella C, Dieci R, Gardini L (2005) The dynamic interaction of speculation and diversification. Appl Math Finance 12(1):17-52. https://doi.org/10.1080/1350486042000260072

Day RH, Huang W (1990) Bulls, bears and market sheep. J Econ Behav Organ 14(3):299-329

Franke R, Westerhoff F (2012) Structural stochastic volatility in asset pricing dynamics: Estimation and model contest. J Econ Dyn Control 36(8):1193-1211. https://doi.org/10.1016/j.jedc.2011.10.004 (quantifying and Understanding Dysfunctions in Financial Markets)

Gaunersdorfer A, Hommes C (2007) Long memory in economics. Springer, Berlin, pp 265-288

Huang W, Day R (1993) Chaotically switching bear and bull markets: the derivation of stock price distributions from behavior rules. In: Day R, Chen P (eds) Nonlinear dynamics and evolutionary economics. Oxford University Press, Oxford

Huang W, Zheng H, Chia WM (2010) Financial crises and interacting heterogeneous agents. J Econ Dyn Control 34(6):1105-1122. https://doi.org/10.1016/j.jedc.2010.01.013

Jungeilges J, Ryazanova T, Mitrofanova A, Popova I (2018) Sensitivity analysis of consumption cycles. Chaos 28(5):055905. https://doi.org/10.1063/1.5024033

Jungeilges J, Maklakova E, Perevalova T (2021) Asset price dynamics in a bull and bear market. Struct Change Econ Dynamics 56(3):117-128

Lux T (1995) Herd behaviour, bubbles and crashes. Econ J 105(431):881-896. http://www.jstor.org/stable/ 2235156

Mil'shtein G, Ryashko L (1995) The first approximation in the quasipotential problem of stability of nondegenerate systems with random perturbations. J Appl Math Mech 59(1):47-56

Nasyrova VM, Ryashko L, Tsvetkov I (2019) Stochastic oscillations in a neuron model with two-dimensional map. In: Volkovich V, Zvonarev S, Kashin I, Smirnov A, Narkhov E (eds) Physics, technologies and innovation, PTI 2019, American Institute of Physics Inc., United States, AIP Conference Proceedings. https://doi.org/10.1063/1.5134294

Panchuk A, Sushko I, Westerhoff F (2018) A financial market model with two discontinuities: bifurcation structures in the chaotic domain. Chaos 28:055908. https://doi.org/10.1063/1.5024382

Schmitt N, Westerhoff F (2017) On the bimodality of the distribution of the S\&P 500's distortion: Empirical evidence and theoretical explanations. J Econ Dyn Control 80:34-53. https://doi.org/10.1016/j.jedc. 2017.05.002

Sushko I, Tramontana F, Westerhoff F, Avrutin V (2015) Symmetry breaking in a bull and bear financial market model. Chaos Solitons Fractals 79:57-72. https://doi.org/10.1016/j.chaos.2015.03.013, http:// www.sciencedirect.com/science/article/pii/S0960077915000946, proceedings of the MDEF (Modelli Dinamici in Economia e Finanza - Dynamic Models in Economics and Finance) Workshop, Urbino 18th-20th September 2014

Sushko I, Gardini L, Avrutin V (2016) Nonsmooth one-dimensional maps: some basic concepts and definitions. J Differ Equ Appl 22(12):1816-1870. https://doi.org/10.1080/10236198.2016.1248426

Tramontana F, Gardini L, Dieci R, Westerhoff F (2009) The emergence of bull and bear dynamics in a nonlinear model of interacting markets. Discrete Dyn Nat Soc. https://doi.org/10.1155/2009/310471

Tramontana F, Westerhoff F, Gardini L (2010) On the complicated price dynamics of a simple onedimensional discontinuous financial market model with heterogeneous interacting traders. J Econ Behav Organ 74(3):187-205. https://doi.org/10.1016/j.jebo.2010.02.008

Tramontana F, Gardini L, Westerhoff F (2011) Heterogeneous speculators and asset price dynamics: Further results from a one-dimensional discontinuous piecewise-linear map. Comput Econ 38(3):329. https:// doi.org/10.1007/s10614-011-9284-9

Tramontana F, Westerhoff F, Gardini L (2013) The bull and bear market model of Huang and Day: some extensions and new results. J Econ Dyn Control 37(11):2351-2370. https://doi.org/10.1016/j.jedc. 2013.06.005 
Tramontana F, Westerhoff F, Gardini L (2014) One-dimensional maps with two discontinuity points and three linear branches: mathematical lessons for understanding the dynamics of financial markets. Decis Econ Finance. https://doi.org/10.1007/s10203-013-0145-y

Tramontana F, Westerhoff F, Gardini L (2015) A simple financial market model with chartists and fundamentalists: Market entry levels and discontinuities. Math Comput Simul 108:16-40. https://doi.org/ 10.1016/j.matcom.2013.06.002

Publisher's Note Springer Nature remains neutral with regard to jurisdictional claims in published maps and institutional affiliations. 